\title{
SOCIEDAD Y CRISIS DE VALORES: INTERROGANTES Y RESPUESTAS DESDE LA BIOÉTICA Y LA BIOJURÍDICA
}

Ronald Cárdenas Krenz*

Presentado: 16.07 .2014

Aprobado: 08.08.2014

\section{RESUMEN}

El artículo constituye una reflexión respecto al mundo actual desde la perspectiva de la Bioética y el Derecho, teniendo en cuenta la importancia de una aproximación ética, frente a la cosificación de la vida humana, diversas situaciones nuevas vinculadas con el desarrollo tecnológico y la crisis de valores de nuestro tiempo, poniendo énfasis en la necesidad de una visión humanista para enfrentarlos.

\section{PALABRAS CLAVE}

Bioética - Autonomía - Tecnociencia - Biojurídica

\section{ABSTRACT}

The article is a reflection on the current world from the perspective of Bioethics and Law, taking into account the importance of an ethical approach considering the commodification of human life, several new technology-related developments, and value crisis of our times, emphasizing on the need for a humanistic approach to address them.

\section{KEY WORDS}

Bioethics - Autonomy - Techno-science - Biolaw

\section{INTRODUCCIÓN}

La Bioética constituye, sin duda, uno de los temas fundamentales de nuestro tiempo. En sus predios convergen tanto la inquie- tud científica como la preocupación social; la reflexión intelectual como la innovación tecnológica ${ }^{1}$; lo ético como lo jurídico, lo individual como lo colectivo, lo reflexivo como lo práctico.
Podemos definirla diciendo que: "La bioética supone un intento de conseguir un enfoque secular, interdisciplinario, prospectivo, global y sistemático, de todas las cuestiones

Abogado y Magister en Derecho Civil y Comercial. Profesor de la Universidad Femenina del Sagrado Corazón, Universidad de Lima y ESAN. Profesor invitado de la Universidad César Vallejo (Trujillo) y de la Maestría en Bioética y Biojurídica de la Universidad Santo Toribio de Mogrovejo. Miembro de la Academia de Derecho y Ciencias Sociales de Córdoba. Past Decano de la Facultad de Derecho de la UNIFE. Ha sido Superintendente Nacional de la SUNARP, Presidente del Consejo del Notariado y Miembro del Comité de Bioética de la Facultad de Biología de la Universidad Nacional Mayor de San Marcos. Co-fundador en el Perú de la Cátedra UNESCO de Bioética y Biojurídica. Considerado por UNESCO en su relación de expertos en ética a nivel mundial: Global Ethics Observatory: Who's Who in Ethics.

1 Así, para Garza-Garza: "La bioética se centra en el estudio de los problemas éticos, que plantea el desarrollo de las diferentes ciencias y tecnologías que pueden aplicarse, incluir o modificar la vida humana". (Garza-Garza, R. Bioética. "La toma de decisiones en situaciones difíciles”. México: Editorial Trillas. Citado por Fajardo Dolci, Germán E. y Héctor Aguirre Gas, 2000. Preceptos éticos y legales de la práctica médica. México: Corporativo Intermédica, S.A. de C.V., p. IX). 
éticas que conciernen a la investigación sobre el ser humano $y$, en especial, a la biología, la medicina y la atención en salud"2.

Frente al frenesí con que nos solemos entregar a las novedades y a las tentaciones de la tecnología y de la investigación científica, es importante asumir una actitud fronética que nos haga detenernos a pensar en qué es lo que estamos haciendo, qué es lo que queremos hacer y si estamos siguiendo el camino correcto para alcanzarlo.

Es cierto que el derecho va por lo general detrás de las nuevas circunstancias pero es que, justamente, detrás de nuestros ímpetus efervescentes, debe venir siempre la razón para hacernos medir con cautela nuestros actos, tomar conciencia de ellos e intentar adoptar las mejores decisiones ${ }^{3}$.
La Bioética está lejos de ser un mero ejercicio teórico o filosófico; al contrario, es una disciplina eminentemente práctica $y$, por ello, es importante el estudio de casos para cualquier debate sobre la materia para, a partir de allí, poder hacer un análisis reflexivo más completo.

\section{LA COSIFICACIÓN DE LA VIDA HUMANA}

Esta necesidad reflexiva se torna más importante si tenemos en cuenta que vivimos hoy en día, como dice Julián Marías, una renuncia a entender, que ha hecho que el hombre deje de hacerse preguntas, generando con ello el que asuma una actitud en que no se ve como persona responsable, con dudas, problemas, fines, deberes ${ }^{4}$. Peor todavía, el ser humano parece no sólo que ha perdido sus valores, sino que también se ha puesto precio a sí mismo, se ha vuelto un producto más del mercado, como lo evidencia, por ejemplo, el hecho de asomarnos cada vez más a los límites del genismo, jóvenes mujeres que subastan su virginidad por internet, o personas capaces de venderse a sí mismas, a niños o hasta a sus propios hijos ${ }^{5}$.

Todo este panorama se agrava cuando vemos que, como dice Serrano, "la tendencia es a presentar los valores como tabúes y las exigencias de la dignidad humana como afirmaciones opinables y fundamentalmente individuales, esto es, sin trascendencia social objetiva"6.

Deslizados hacia la cosificación de la vida humana, como señala María Cruz Díaz de Terán Velas $\mathrm{CO}^{7}$, esto se expresa en casos como el tráfico de órganos, el denominado "turismo sexual", la existencia de páginas web en las que se subastan óvulos y

2 León Francisco (2011). La promoción de la dignidad de la persona en el ámbito biomédico. Madrid: Ediciones Palabra, p. 13.

3 “Cierto es que algunas de las acusaciones recibidas por el Derecho son francamente rechazables. Me detendré en la primera obviedad que hay que soportar en cualquier congreso médico. La respuesta jurídica es siempre posterior, incluso retrasada respecto a la realidad y muchas veces con normas viejas. En este punto quizás conviniera recordar que este actuar sobre una realidad ya producida es una característica del propio Derecho y que las alternativas no son ni mucho menos mejores. Por otro lado, la readaptación de las leyes y de los viejos conceptos es una vieja tradición del Derecho con inmejorables resultados (...). En este punto, sería deseable que unos no hiciéramos puntualizaciones infundadas sobre elementos científicos, si otros no hiciesen simplificaciones jurídicas”. (Serrano Ruiz-Calderón, José Miguel, 2004, "Bioética, genética y derecho". En: Genética y Derecho. Cuadernos de Derecho Judicial VI-2004. Madrid: Consejo General del Poder Judicial, p. 33).

4 Marías, Julián (1993). Razón de la Filosofía. Madrid: Alianza Editorial, p. 46-47. A ello se agrega "la atomización de la vida, la pérdida de sus formas, el aturdimiento. Una desorientación acaso más profunda que ninguna otra, porque el hombre que la padece no sabe que está desorientado. Se deja llevar, y ni siquiera se da cuenta que está perdido" (Ibid., p. 52).

5 Una noticia en octubre del 2013, daba cuenta, por ejemplo, de una pareja de desempleados en Shangai (China) que vendió a su tercera hija por internet para comprar un iPhone y otros objetos de lujo (http://www.tecnoxplora.com/internet/ pareja-china-vende-hija-internet-comprar-iphone_2013102000049.html); en enero del 2012, se conoció la noticia de un hombre en Arabia Saudita que ponía a la venta a su hijo en Facebook al mejor postor por US\$20 millones, alegando que lo hacía para ofrecer "una vida digna a su madre y su hermana en lugar de vivir en la pobreza" (http://peru21. pe/2012/01/09/mundo/pone-venta-su-hijo-facebook-2006742); en octubre del 2014, en Salta (Argentina) fue detenido un individuo que por medio de Facebook había publicado la foto de una menor de edad ofreciendo en venta sus "servicios" para realizar servicios sexuales (http://www.larepublica.pe/29-10-2014/anuncio-en-facebook-de-nina-en-venta-causaindignacion-en-argentina); por la misma fecha, se difundió el caso de una muchacha de 19 años -Ju Peng- anunciando sus servicios sexuales para costear su viaje por distintos lugares de su país (http://www.larepublica.pe/28-10-2014/ facebook-joven-china-ofrece-sus-servicios-sexuales-para-financiar-su-excursion).

6 Serrano Ruiz-Calderón, José Miguel (2004). Op. cit., p. 29.

7 Díaz de Terán Velasco, María Cruz (2005). Derecho y nueva eugenesia. Un estudio desde la ley 35/88 de 22 de noviembre de Técnicas de Reproducción Asistida. Pamplona: Ediciones Universidad de Navarra S.A., p. 79. 
embriones al mejor postor ${ }^{8}$, el tráfico de niños ${ }^{9} \mathrm{o}$ a que, como parte de esta tendencia deshumanizadora, a los recién nacidos producto de abortos que llegaron a sobrevivir, se les deje morir al amparo de la ley en España, alegando simplemente que "no son viables"10.

Todo ello sin contar con casos más anecdóticos en los que el cuerpo puede terminar reducido a la condición de un panel publicitario, como en el caso de Karl Smith, en Salt Lake City (EE.UU), quien en junio de 2.005, aceptó llevar en la frente un tatuaje con el nombre de "GoldenPalace.com", con letras de un tamaño de $2.5 \mathrm{cms}$., a cambio de la suma de 10.000 dólares, la misma que -según dijo- utilizaría para darle educación privada a su hijo de 11 años ${ }^{11}$.

Ya antes Julián Marías hablaba de un mundo caracterizado por la invasión de las cosas ${ }^{12}$, y su derivación en la cosificación de nuestra propia existencia, manifestada, por ejemplo, en el tráfico ilícito de drogas, el tratamiento del suicidio o la permisividad del aborto, tema este último en el que la vida del feto, sorprendentemente, llega a ser reconocida por algunos apenas como un "cuasiderecho"13.

Todo esto sin contar con las amenazas para la humanidad que asoman bajo el "transhumanismo", que revela una mercanti- lización del cuerpo humano, reducido a un objeto de mercado destinado a su progresivo mejoramiento industrial. Como lo dijera el profesor español Nicolás Jouve en el XVIII "Congreso Internacional de Ciencia, Humanismo y Posthumanismos", organizado por la Universidad Libre Internacional de las Américas, en julio del 2013, el transhumanismo supone una deshumanización que hará que por medio de la tecnología se capacite a unos cuantos seres seleccionados en función de criterios espurios en detrimento del resto de la humanidad, creando castas que lleven a separar unos seres humanos de otros con criterios eugenésicos.

\section{Algunas Cuestiones Vincula- DAS CON LA REPRODUCCIÓN ASIS- TIDA Y OTROS TEMAS VINCULADOS CON LA VIDA MISMA}

En materia de reproducción asistida, nos encontramos hoy por hoy con infinidad de casos polémicos, que llevan a diversas reflexiones éticas, jurídicas y existenciales respecto a nuestra propia entidad y diversas cuestiones sociales, incluyendo el de mujeres que dan a luz a su propio nieto (como Elizabeth Sales que dio a luz a su nieta en julio de 2004); o el de Adriana Iliescu que, habiendo dado a luz una niña a los 67 años en enero del 2005 en Rumanía, se convirtió en la madre de mayor edad en el mundo ${ }^{14}$, reanudando el tema de si debe haber una edad para la "jubilación" materna y el debate entre un supuesto derecho reproductivo y el principio del interés superior del niño.

Sobre este último tema, es interesante mencionar también el caso de Susan Tollefsen, quien en el 2008, a los 57 años de edad, fuera una de las mamás primerizas de mayor edad en Gran Bretaña, no pasando mucho tiempo para que reconociera que habían tenido razón los que la criticaron y proponer que debería ponerse un límite de 50 años para las mujeres que deseen recurrir a una fecundación in vitro para poder convertirse en madre.

Otro caso es el de Carmen Bousada de Lara, que fue mamá de un par de gemelos a los 67 años en España, gracias a la fecundación in vitro, en el 2006, siendo tildada por muchos como egoísta. Los niños terminaron quedando huérfanos tan solo 3 años después, a causa de un cáncer que acabó con la vida de ella.

Al lado de estos temas nuevos para el Derecho, existen viejos debates que se han renovado, como los referentes no solo al aborto sino también a la eutanasia, en los que, como dice Dworkin, "las discusiones nunca han sido tan apasionadas y abiertas, las opiniones nunca tan divididas y la controversia

Sobre subasta de óvulos y espermatozoides, véase, por ejemplo la página en internet: www.ronharris.com.

9 Por ejemplo, en julio de 2004, diversos medios daban cuenta de la noticia de una red en París que los vendía a 5,000 ó 6,000 euros según fuera niño o niña.

10 Vila Coro, María Dolores (2003). La bioética en la encrucijada. Madrid: Dykinson, p. 35.

11 Diario El Comercio, Lima: edición del 01.07.05, p. A14.

12 Marías, Julián (1993). Op. cit., p. 45 y sgtes.

13 Vásquez, Rodolfo (2004). Del aborto a la clonación. Principios de una bioética liberal. México: Fondo de Cultura Económica, p. 56.

14 Diario La República, Lima: edición del 17.01.05, p. 27. 
sobre una de estas elecciones nunca ha estado tan íntimamente relacionada con la controversia sobre la otra, como ocurre en estos momentos en Estados Unidos y Europa”"15.

\section{Sobre la relación entre Bió́tica y DeRECHo}

Para abordar estos y otros problemas fundamentales, que vinculan al Derecho y a la Bioética, es importante profundizar en sus contenidos, a efectos de poder resolver muchas de las interrogantes que hoy se presentan, que pronto se formularán o que deberíamos estarnos ya planteando. Empero, antes que esperar encontrar en la Bioética una respuesta concreta a cada una de las cuestiones que afloran, debe dejarse constancia y valorarse su naturaleza dialógi$\mathrm{ca}^{16}$.

Puede decirse aquí que la importancia de la relación entre bioética y derecho: "radica también en un elemento fundamental, de carácter universal, como es que los avances biomédicos afectan a cuestiones sociales de primer nivel -relación de parentesco y filiación, usos del cuerpo, tiempo del nacer y del morir-, que venían reguladas jurídicamente y que, tras esos avances, resulta necesario volver a regular y revisar posturas tradicionales del derecho, forzando la promulgación de nuevas normas jurídicas o a la revisión de las ya existentes"17.

No obstante que el término "bioética" fuera introducido en el debate intelectual hace relativamente poco tiempo, a inicios de los 70, por Van Renselaer Potter, profesor de oncología de la Universidad de Wisconsin, a través de un artículo y luego a través del libro "Bioética: Un puente hacia el futuro", hoy, apenas unas décadas después, ha tomado una relevancia notable y acaso sea la disciplina que más rápidamente se ha desarrollado en los últimos tiempos.

La Bioética -como señala María Jesús Goikoetxea Iturre- gui ${ }^{18}$ - ofrece un método para el análisis y la resolución de los conflictos éticos que surgen en la práctica sanitaria (y no sólo en ella), de modo que sus resultados puedan traducirse en actos morales. En ese sentido, un objetivo prioritario de una metodología en bioética, más que la decisión a tomar, es el mecanismo para llegar a la misma, entendiendo el conflicto siguiendo a Diego Gracia- como un dilema respecto al cual hay una respuesta correcta que es universal y absoluta, que aspiramos alcanzar ${ }^{19}$.

Para el tratamiento de los temas que se mencionan y, en general, los que tienen que ver con la bioética, se pone de relieve el papel de la moral, la cual, como señala con acierto María Dolores Vila-Coro:

“... no solo sirve de cimiento a las leyes sino que presta el gran servicio que supone clarificar las normas que el Derecho no alcanza a definir. Así, en los ordenamientos ju-

15 Señala Dworkin que: “La discusión sobre la eutanasia ha saltado de pronto a las primeras páginas de los periódicos. Los médicos empiezan ahora a admitir abiertamente lo que la profesión mantuvo antes en secreto: que los médicos a veces matan a los pacientes que piden morir o que les ayudan a que se quiten la vida”. (Dworkin, Ronald, 1998. El Dominio de la Vida. Una discusión acerca del aborto, la eutanasia y la libertad individual. Barcelona: Editorial Ariel, p. 9).

16 “... si realmente creemos en la Bioética, debemos aceptar que estamos inmersos en un proceso de discernimiento y descubrimiento respecto a todo aquello que no aceptamos de la opinión ajena, y que requiere mucho más aún de la escucha mutua, no condenatoria sino abierta al diálogo, desde la humildad intelectual” (Abel i Fabre, Francesc, 2012, "La Bioética actual: Problemas y Perspectivas". En: Feito Grande, Lyvia y Tomás Domingo Moratalia (editores). Investigación en Bioética. Madrid: Universidad Rey Juan Carlos - Dykinson, p. 33).

17 Terribas I Sala, Núria (2012). “Bioética y Derecho”. En: Feito Grande, Lydia y Tomás Domigo-Moratalia (Editores). Op. cit., p. 219.

18 Goikoetxea Iturregui, María Jesús. Las decisiones éticas en situaciones sanitarias conflictivas (2003). En: Vélez, Juan, S.J. Bioéticas para el siglo XXI. 30 años de Bioética (1970-2000). Op. cit., p. 173.

19 Sobre los métodos aplicables en Bioética, Goikoetxea, menciona los siguientes:

Métodos principalistas o deontológicos: Entienden que hay una serie de principios morales y absolutos que deben cumplirse siempre. Carácter deductivo; consideran que existen acciones intrínsecamente malas.

Métodos consecuencialistas o teleológicos: Vinculados con el utilitarismo, consideran que la solución o respuesta moralmente más aceptable es aquella que maximiza las consecuencias positivas para los sujetos implicados en la decisión. No consideran que el proceso o los medios sean lo que determine la bondad o corrección moral del fin, sino que es el fin el que en todo caso podrá justificar moralmente los medios o procedimientos.

Métodos o procedimientos mixtos: Consideran que hay un nivel en el razonamiento moral que enuncia las responsabilidades generales y que por tanto puede materializarse en principios exigibles prima facie a cualquier sujeto, y otro nivel que responde a las obligaciones individuales en cada circunstancia particular que puede justificar excepciones a los principios generales.

(Goikoetxea Iturregui, María Jesús. (2003). Op. cit., p. 173). 
rídicos de nuestra área occidental nos encontramos con estándares como el honrado comerciante, la diligencia del buen padre de familia, el arbitrio del hombre bueno... Las apelaciones directas a la moral en los Códigos civiles son constantes: al declarar la nulidad de los contratos contrarios a la moral no es preciso explicar demasiado lo que esto significa. La buena fe está en la base y es el fundamento de nuestro orden jurídico, especialmente del derecho mercantil y civil ¿cómo sería posible el tráfico jurídico si no es sobre la base de la buena fe y de que se va a obrar de acuerdo con la moral? Imagine el lector si hubiera que preguntar a cada ciudadano cuando alquila un piso, compra un coche a plazos, o recibe un préstamo, a qué moral o ética se encuentra adscrito. Esta aceptación de hecho de una moral común es una prueba más de la objetividad y universalidad de la ley moral"20.

En adición a ello, como dice Roberto Andorno, la bioética no puede quedar en el ámbito individual, pues se volvería una simple casuística ${ }^{21}$.

Así, resulta evidente que debe protegerse el derecho a la autonomía, el derecho al consentimiento informado, el derecho a saber respecto a hechos que conciernen a uno mismo, los cuales son importantes para poder ejercer correcta y responsa- blemente nuestra libertad; sin embargo, como muestra de los obstáculos que deben sortearse en este propósito, nos encontramos con decisiones sorprendentes como la de la Corte Suprema de los Estados Unidos en 1993, en el proceso de un centro de salud reproductiva contra el municipio de Akron Ohio, que consideró que "mostrarle a una mujer que solicita un aborto una fotografía de un embrión, de la edad del que ella quería destruir, era no sólo un medio ilegítimo de ofrecer información para el consentimiento al aborto, sino que constituía un atentado a la libertad; equivalía a un desfile de atrocidades, una muestra de tortura ideológica"22.

Conforme a ese fallo (que en el fondo reconoce al aborto como un desfile de atrocidades), la verdad puede torturar y ser peligrosa para la libertad, cuando lo cierto es que la libertad, en tanto implica la posibilidad de tomar decisiones, requiere de la verdad y de la información como condiciones esenciales para su adecuado ejercicio. $\mathrm{Si}$ en un país el aborto está legalizado -independientemente de que estemos o no de acuerdo con ello-, será licito que una clínica lo permita, pero su obligación mínima es informarle al paciente en qué consiste el aborto al que quiere someterse, $a$ fin de que la mujer interesada pueda prestar su consentimiento en forma informada, tal como se aprobara en Texas en donde, en el 2011, se estableció como obligatorio que las mujeres que vayan a abortar escuchen una descripción detallada del feto hecha por un médico y esperen luego 24 horas para someterse al aborto; la descripción médica no es obligatoria en el caso de víctimas de violación, a las menores y a aquellas madres cuyo feto tenga alguna malformación. En Oklahoma, en cambio, en una ley similar dada en el 2010, se estableció expresamente que no habría excepciones en caso de incesto o violación ${ }^{23}$.

\section{Humanidad, tecnología Y CUESTIONES PENDIENTES}

Por otro lado, volviendo a las implicancias del desarrollo de las nuevas tecnologías, no debe dejarse de tener presente que la cuestión ética se torna más compleja por el hecho de que las biotecnologías ya no operan únicamente sobre el mundo exterior, sino sobre el propio ser humano, lo que le otorga a la biología una trascendencia e impacto jamás alcanzado; hoy, a través de un microscopio, estudiando los genes, podemos conocer mejor de nosotros mismos que a través de un telescopio hurgando los astros.

No es inusual advertir que, a lo largo de la historia, como ahora (aunque no tanto a un ritmo tan rápido) los avances de la tecnología hayan despertado no solo satisfacción sino también preocupación, pero se ha dicho atinadamente que la ciencia es neutra, no es ni buena ni mala,

Vila-Coro, María Dolores (2003). Op. cit., p. 35.

Andorno, Roberto (1998). Bioética y dignidad de la persona. Madrid: Editorial Tecnos, p. 34.

22 Herranz, G. (1991). “Aspectos médico-farmacéuticos del embrión humano”. En: El inicio de la Vida, B.A.C. Madrid, 1991, p. 191, citado por Vila-Coro, María Dolores (2003), Op. cit., p. 146.

23 Diario El País, edición digital. Madrid: edición del 11.05.2011. 
ya que lo importante es el uso que se haga de ella. Empero, dados los niveles alcanzados por la tecnología actual, el asunto es más delicado, a tal punto que, como señala Juan Ramón Capella, "es ingenuo suponer que la peligrosidad del instrumental tecnocientífico depende de los sujetos en cuyas manos esté, o, lo que es lo mismo, que la ciencia actual sólo es peligrosa en función de las intenciones de los sujetos que la usan. Las intenciones de los sujetos han sido potencialmente peligrosas desde la invención del garrote, pero ahora nos hallamos ante un problema distinto" 24 .

Existe entonces una peligrosidad ontológica, de la tecnociencia contemporánea, teniendo en cuenta que el riesgo ya no solo se trata de la amenaza a una persona o grupo de personas, sino a la humanidad como tal.

Claro que, como afirma el mismo Capella, obviamente no se puede renunciar a la ciencia, pero probablemente el amparo frente a su peligrosidad no vendrá de ella misma (recuérdese a Heráclito: "Haber aprendido muchas cosas no ayuda a tener entendimiento"); por lo que debe tenerse en cuenta aspec- tos morales y políticos ${ }^{25}$, a los que -en nuestra opinión- habría que agregar la consideración de aspectos sociales y jurídicos.

La preocupación por estos temas no constituye mera especulación, sino que está vinculada con una ética de carácter preventivo, siendo conveniente adoptar acciones de este tipo antes que medidas que evadan u oculten los problemas de fondo, o que aborden los efectos mas no las causas. Así como frente a la obesidad infantil la solución no es mandar a hacer tallas de ropa más grande para los menores, tampoco puede plantearse como política frente al embarazo adolescente el simplemente repartir preservativos o regalar bonos en hoteles ${ }^{26}$.

En la misma línea de una actitud phronética que planteamos, es importante mantener una racionalidad adecuada en la experimentación y tratamiento científico; diversas situaciones que se vienen dando ${ }^{27}$ nos llevan a enfatizar la necesidad, de un racionalismo humanista, aun cuando la expresión pueda ser redundante, pero que resulta útil para resaltar la imprescindibilidad de una visión humanista al tratar estos temas.
La realidad descrita va aparejada con otra característica que define a nuestro tiempo: la existencia de una actitud hedonista e irresponsable ${ }^{28}$, en cuyo contexto la satisfacción personal tiene primacía aun cuando ella implique ejercer un uso instrumental del otro. Ejemplo de ello ${ }^{29}$ es el caso de una francesa, de 62 años, que, en el 2000 , fue al médico porque quería tener un hijo por fecundación in vitro, utilizando semen y óvulos donados; aunque ella no le contó el detalle al médico, de que el esperma era nada menos que de su hermano. El galeno rechazó tratarle, en gran parte porque tenía sospechas acerca de su estado psicológico. Entonces, la mujer y su hermano se fueron a Los Ángeles, en donde, ocultando también el hecho que el donante era su hermano, obtuvo el tratamiento de la fecundación in vitro y dio a luz a un niño. Simultáneamente, como una especie de salvaguarda, una madre sustituta, la donante del óvulo, fue inseminada con el semen del hermano y dio también a luz a un bebé, en este caso una niña. El caso provocó que en Francia se hablara con escándalo de un "incesto social"30.

24 Capella, Juan Ramón (2004). Elementos de análisis jurídico. 3ª . Edición. Madrid: Editorial Trotta, S.A., p. 41.

25 Ibid., p. 43.

26 Refiere Vila-Coro que, en España, como parte de una campaña del Ministerio de Sanidad y el Consejo de la Juventud consistente en el uso del preservativo para la prevención del SIDA, se incluyeron dinámicas grupales de carácter lúdico que incluyeron en pasadas ediciones la degustación de preservativos de sabor para niños y niños de doce años en clases de colegios públicos (Vila-Coro, María Dolores, 2003, Op. cit., p. 68).

27 Jacques Testart señala que en los casos de fertilización asistida, en promedio se obtienen diez óvulos por mujer, habiendo visto casos en que se ha producido hasta ochenta (Testart, Jacques y Christian Godin, 2002, El racismo del gen. Buenos Aires: Fondo de Cultura Económica, p. 18).

28 Afirma Christian Godin: "El eugenismo se sustenta en una ilusión terriblemente peligrosa, una ilusión que también vehiculiza: la de creer que el bienestar (no hablemos de la felicidad) reside solamente en la salud física. Como afirma el psicólogo Ehremberg, una sociedad cuyo horizonte es el "mejor estar" sólo puede ser una sociedad del malestar" (Testart, Jacques y Christian Godin. Op. cit., p. 55).

29 Citado por Warnock, Mary. (2004). Fabricando bebés. ¿Existe un derecho a tener hijos? Barcelona: Editorial Gedisa, p. 57-58.

30 Agrega la mencionada autora que "En cualquier caso, en Francia es ilegal dar asistencia para la reproducción a una mujer posmenopáusica, al estar basada la ley en el concepto del bien del niño, así como en un sentimiento ampliamente compartido expresado en la declaración de que "hay un tiempo para ser madres y un tiempo para ser abuela", y en que este 
En este tipo de casos, siguiendo a Andorno ${ }^{31}$, nos encontramos ante un conflicto entre una ética relativista (o subjetivista) y una ética objetivista (o personalista). Para la primera, los principios éticos no son verdaderos ni falsos, sino simple resultado del acuerdo o la elección arbitraria, no habiendo acción intrínsecamente mala. Para la segunda, en cambio, es posible acceder al conocimiento del bien por medio de la razón.

Dentro del relativismo -agrega el mencionado autor-, el no cognotivismo parte de la imposibilidad de trascender lo puramente fenoménico y rechaza la capacidad de la razón para acceder a la verdad en lo moral; la elección ética no tiene que ver con ella por no ser verificable empíricamente; este escepticismo hace que Hume atribuya al sentimiento y no a la razón el papel de determinar la bondad de las acciones humanas. Por su lado, el neoutilitarismo busca maximizar el placer y minimizar el dolor; el cálculo del coste social es la regla moral válida para todos. A su vez, el neocontractualismo hace coincidir el bien moral con el acuerdo entre los sujetos, se trata de una decisión colectiva, sin una sustancia predeterminada y más bien con un contenido variable.

Frente a ello -continúa Andorno-, el pensamiento objetivista estima que el bien no es resultado de una elección arbitraria del hombre, sea individual o colectiva; hay acciones que son intrínsecamente malas. "El bien no es construido, sino reconocido. La razón humana es juzgada capaz de distinguir lo que contribuye al bien de la persona de aquello que puede dañarla"; la persona es el fin de todas las instituciones sociales, políticas, económicas, etc.

No cabe duda, por tanto, de la necesidad de poner de relieve la trascendencia de la bioética, en cuyo contenido, "El punto común de los nuevos interrogantes es el valor del hombre en su corporeidad frente a los desarrollos biomédicos. Por ello, puede afirmarse, en una primera aproximación, que la reflexión bioética no hace más que retomar el cuestionamiento eterno del hombre sobre sí mismo y su dignidad, adaptado a las circunstancias actuales". En este contexto, por su misma complejidad y alcances, la bioética supone “... una aproximación interdisciplinaria, prospectiva, global y sistemática a los nuevos dilemas" 32 .

La libertad no tiene como límite tan solo la libertad del otro, sino también nuestra propia humanidad, a la que no podemos renunciar, pues sin esa consideración la libertad -que no es mero arbitrio- no tendría sentido.

El desarrollo de la bioética permite ponderar mejor los alcances del principio jurídico de la autonomía de la voluntad; su relación con el principio de autonomía, desarrollado ampliamente en la Bioética, interrela- cionado con otros principios de la misma -como los de la prudencia, el respeto a la dignidad, la justicia, la beneficencia, etc.-, posibilitan para el Derecho un tratamiento más adecuado a fin de abordar cuestiones tan delicadas como las planteadas en el presente artículo.

Los tiempos actuales demandan una revisión de los fundamentos mismos de nuestra propia existencia, a partir de una reflexión ética ciertamente, cuyas conclusiones deben trascender en propuestas jurídicas concretas. Se trata de una tarea urgente, compleja pero necesaria, a la que sin duda debemos llegar, apurados por el avance tecnológico y la demanda social; la duda más bien es saber si podremos llegar a esa reflexión a tiempo.

\section{REFERENCIAS BIBLIOGRÁFICAS}

Abel i Fabre, Francesc. (2012). La Bioética actual: Problemas y Perspectivas. En: Feito Grande, Lyvia y Tomás Domingo Moratalia (editores). Investigación en Bioética. Madrid: Universidad Rey Juan Carlos - Dykinson.

Andorno, Roberto (1998). Bioética y dignidad de la persona. Madrid: Editorial Tecnos.

Capella, Juan Ramón (2004). Elementos de análisis jurídico. $3^{a}$. Edición. Madrid: Editorial Trotta, S.A.

Dworkin, Ronald (1998). El Dominio de la Vida. Una dis-

tipo de nacimientos tardíos contravienen la ley del sentido común. En este caso particular originado en Francia, había una historia de espantosas discordias familiares sobre la herencia de una propiedad, y parece que el motivo de haber tenido los dos bebés fue que la herencia no se repartiese con sus familiares cercanos" (Ibid., p. 58).

31 Andorno, Roberto (1998). Op. cit., p. 28 - 30.

32 Ibid., p. 12 - 13. 
cusión acerca del aborto, la eutanasia y la libertad individual. Barcelona: Editorial Ariel.

Fajardo Dolci, Germán E. y Héctor Aguirre Gas (2000). Preceptos éticos y legales de la práctica médica. México: Corporativo Intermédica, S.A. de C.V.

Goikoetxea Iturregui, María Jesús (2000). "Las decisiones éticas en situaciones sanitarias conflictivas". En: Vélez, Juan, S.J. Bioéticas para el siglo XXI. 30 años de Bioética (1970-2000) Bilbao: Universidad de Deusto.

Gracia, Diego (2003). "Fundamentaciones de la Bioética". En: Vélez, Juan S.J. (coord.). Bioética para el siglo XXI.
30 años de Bioética (19702000). Bilbao: Universidad de Deusto.

León Correa Francisco (2011). La promoción de la dignidad de la persona en el ámbito biomédico. Madrid: Ediciones Palabra.

Marías, Julián (1993). Razón de la Filosofía. Madrid: Alianza Editorial.

Serrano Ruiz-Calderón, José Miguel (2004). "Bioética, genética y derecho". En: Genética y Derecho. Cuadernos de Derecho Judicial VI-2004. Madrid: Consejo General del Poder Judicial.

Terribas I Sala, Núria. (2012). "Bioética y Derecho". En: Feito Grande, Lydia y Tomás
Domigo-Moratalia (Editores). Investigación en Bioética. Madrid: Universidad Rey Juan Carlos - Dykinson.

Testart, Jacques y Christian Godin (2002). El racismo del gen. Buenos Aires: Fondo de Cultura Económica.

Vásquez, Rodolfo (2004). Del aborto a la clonación. Principios de una bioética liberal. México: Fondo de Cultura Económica.

Vila-Coro, María Dolores (1995). Introducción a la Biojurídica. Madrid: Universidad Complutense.

Warnock, Mary (2004). Fabricando bebés. ¿Existe un derecho a tener hijos? Barcelona: Editorial Gedisa. 